\title{
Highly Safe 100-Wh-class Lithium-ion Battery Using Lithium Bis(trifluoromethanesulfonyl)amide-Tetraethylene Glycol Dimethyl Ether Equimolar Complex-based Quasi-solid-state Electrolyte
}

\section{Atsushi UNEMOTO, ${ }^{a}, *$ Suguru UEDA, ${ }^{a}$ Eiji SEKI, ${ }^{a}$ Masanari ODA, ${ }^{a}$ Jun KAWAJI, ${ }^{a}$ Takefumi OKUMURA, ${ }^{a}$ Yoshiyuki GAMBE, ${ }^{b}$ and Itaru HONMA ${ }^{b}$}

\author{
${ }^{a}$ Research \& Development Group, HITACHI, Ltd., 7-1-1 Omika, Hitachi, Ibaraki 319-1292, Japan \\ b Institute of Multidisciplinary Research for Advanced Materials, Tohoku University, \\ 2-1-1 Katahira, Aoba-ku, Sendai 980-8577, Japan
}

* Corresponding author: atsushi.unemoto.zt@hitachi.com

\begin{abstract}
A highly safe 100 Wh-class laminated lithium ion battery (LIB) was developed. For ensuring safety of the LIB, a liquid electrolyte was quasi-solidified at silica surfaces. For the liquid electrolyte, a solvate ionic liquid (SIL), which is an equimolar complex of lithium bis(trifluoromethanesulfonyl)amide (LiTFSA) and tetraethylene glycol dimethyl ether (G4), Li(G4)TFSA, was used. For enhancing discharge-rate capability, Li(G4)TFSA was diluted by propylene carbonate (PC). Then, for enhancing cycle life, vinylene carbonate (VC) and hexafluorophosphate anion ( $\left.\mathrm{PF}_{6}{ }^{-}\right)$based salt were added for forming an solid-electrolyte interphase (SEI) on the graphite negative electrode and an $\mathrm{AlF}_{3}$ at the surface of the aluminum current collector of the positive electrode, respectively. The assembled LIB exhibited initial discharge capacity of $32 \mathrm{Ah}$ and coulombic efficiency of $76 \%$. Regardless of high energy-type, the developed battery exhibited high discharge capacity of $26.2 \mathrm{Ah}$ at $2 \mathrm{C}$. Its retention ratio of discharge capacity at the 118 th cycle is high, i.e., $96 \%$. The developed LIB (with energy density of $363 \mathrm{Wh} \mathrm{L}^{-1}$ ) generated neither fire nor smoke in a nail-penetration test. These results suggest that the developed LIB has high safety compared to a LIB comprised of a conventional organic liquid electrolyte.
\end{abstract}

(c) The Electrochemical Society of Japan, All rights reserved.

Keywords : High-energy Battery, Quasi-solid-state Electrolyte, Safety, Nail-penetration Test

\section{Introduction}

Lithium-ion batteries (LIBs) are currently used in a broad range of applications, including portable devices and automobiles, because of their high energy density. One of the emerging issues that needs to be addressed is ensuring the safety of LIBs, which is currently problematic from the viewpoint of applications. In general, organic liquid solvents have high vapor pressure and are inflammable; thereby, current LIBs using organic liquid solvents have a risk of catching fire and exploding upon unexpected accidents. ${ }^{1}$ Hence, for large-scale applications, including vehicles, current rechargeable LIB systems require a safety system and a cooling space, which do not directly contribute to energy storage. If the safety of LIBs were improved, the energy-storage system could be simplified, and that simplification would be advantageous in terms of enhancing energy density and cost competitiveness of rechargeable systems. Therefore, a design principle for LIBs that meets the requirement of essential safety is urgently required.

An all-solid-state battery, which uses a solid-state electrolyte and hence is comprised of all solid-state components, would be a possible approach to ensure battery safety. ${ }^{2,3}$ Materials that possess sufficient lithium-ion conductivity as well as electrochemical stability in the voltage range for battery operation are limited to several in number..$^{3-5}$ In addition, to reduce internal resistance and enhance cycle life, it is necessary to develop technologies that allow for a smooth charge-transfer reaction and hinder mutual diffusion of constituent elements across the electrode active material and electrolyte particles in the composite electrode layers. ${ }^{6,7}$

Another approach is to use a room-temperature ionic-liquid (RTIL) electrolyte, which has negligible vapor pressure and nonflammability and, thereby, ensures battery safety. ${ }^{8,9}$ High safety of this class of materials (RTIL) is suggested by ignition tests. ${ }^{26,42}$ As another aspect, it is known that RTILs are quasi-solidified owing to a strong interaction between the oxide surface and RTILs. It was reported that RTILs have a highly ordered structure at a charged sapphire substrate with a few layers. ${ }^{10}$ In another report, it was revealed that the viscosity of RTILs is enhanced just beneath the silica substrate surface by approximately three orders of magnitude compared to that in the bulk. Then, it relaxes within approximately $10 \mathrm{~nm}$ from the substrate surface. ${ }^{11}$ A quasi-solid-state electrolyte (QSE), which is the result of mixing RTILs with oxide nanoparticles, exhibits liquid-like high ionic conductivity as well as solid-like mechanical toughness. ${ }^{12-19}$ On the basis of a design principle exploiting this electrolyte, electrochemical devices such as fuel cells, ${ }^{13}$ dye-sensitized solar cells, ${ }^{14}$ electric double-layer capacitors, ${ }^{15}$ and LIBs ${ }^{16-19}$ have been developed. Since a liquidphase electrolyte is quasi-solidified by mixing with oxide particles, a QSE is expected to hinder leakage of the liquid phase from the electrolyte layer.

Solvate ionic liquids (SILs), which exhibit similar physicochemical properties to RTILs, are attracting particular attention for use as the electrolyte of a LIB. An equimolar mixture of lithium bis(trifluoromethanesulfonyl)amide, LiTFSA, and tetraethylene glycol dimethyl ether (or tetraglyme), G4, namely Li(G4)TFSA, is a typical example of a SIL. ${ }^{20,21} \mathrm{Li}(\mathrm{G} 4)$ TFSA has attractive properties as an electrolyte of a LIB, including excellent thermal stability, lower vapor pressure, and superior electrochemical stability. ${ }^{20,21}$ Repeated operation of a LIB that uses quasi-solid-state Li(G4)TFSA has been demonstrated. ${ }^{22}$ For LIBs that use SILs, intrinsically high concentration of Li-salt in the electrolyte enhances the viscosity of the SILs; thereby, ionic conductivity is suppressed. In addition, high concentration of Li-salt causes high internal 
resistance owing to concentration polarization under current flow. ${ }^{23}$ To solve this problem, Li(glyme)TFSA (glyme $=$ G3 and G4) was diluted with a wide range of solvents, and the stability of $[\mathrm{Li}(\text { glyme })]^{+}$was then investigated. ${ }^{23,24}$ When nonpolar solvents, such as hydrofluoroether (HFE), are chosen for the dilution, $[\mathrm{Li}(\text { glyme })]^{+}$stays as is in the mixture. On the other hand, in polar solvents, like water and propylene carbonate (PC), ligand exchange takes place, resulting in a formation of free-G4. And it was reported that the diluted SILs readily enhance rate capability of LIBs. ${ }^{24,25}$

To ensure both rate capability and safety of a LIB, Li(G4)TFSA must be diluted with a solvent that has comparatively low vapor pressure and high flash point. Accordingly, it is preferable if the resultant electrolyte liquid is quasi-solidified by mixing with solid particles for ensuring the LIB safety by avoiding leakage and lowering the liquid amount required for assembly of the LIB. In the current study, PC was chosen as a low-viscosity diluting medium, and the resultant mixture, i.e., Li(G4)TFSA-PC, was quasi-solidified with silica particles. Although mixing of $\mathrm{Li}(\mathrm{G} 4)$ TFSA with PC causes ligand exchange, ${ }^{23}$ interaction between PC and Li-ions is pronounced; thereby, PC solvent is expected to be less vaporized from the QSE than PC itself. In addition, $\mathrm{PC}$ has a high flash point of approximately $403 \mathrm{~K}^{26,27}$ (in comparison, $414 \mathrm{~K}$ for $\mathrm{G} 4,{ }^{28}$ and 293-303 K for conventional organic liquid electrolytes containing linear carbonates such as $\mathrm{LiPF}_{6}$ in ethylene carbonate:ethyl methyl carbonate $\left.(\mathrm{EC}: \mathrm{EMC})^{29,30}\right)$. Hence, we considered that quasi-solidstate $\mathrm{Li}(\mathrm{G} 4)$ TFSA-PC would be advantageous for ensuring LIB safety.

As explained in the next section, the ion dynamics of the Li(G4)TFSA-PC mixture was investigated, and the composition of the mixture was optimized for use as the electrolyte of a LIB. Then, a high-capacity 100 -Wh-class laminated cell was assembled using the QSE, comprised of Li(G4)TFSA-PC, and its fundamental battery performance was evaluated. In the last section, high safety of the 100-Wh-class LIB was successfully demonstrated via a nailpenetration test.

\section{Experimental}

\subsection{Preparation and characterization of quasi-solid-state electrolyte}

G4 (Merck KGaA, $\geq 99 \%$ ) and PC (Kishida Chemical Co., Ltd., 99.5\%) were used as received. LiTFSA was purchased from $3 \mathrm{M}$, Inc. and purified by annealing at $393 \mathrm{~K}$ for $2 \mathrm{~h}$ in vacuum for removing residual water. The resultant powder contained only $40 \mathrm{ppm}$ of water, which was analyzed by a neutron magnetic resonance (NMR, ECA-500, JEOL Resonance, Inc.) spectroscope. G4 and LiTFSA were mixed in a molar ratio of $1: 1$, forming Li(G4)TFSA. Li(G4)TFSA was further mixed with PC in the desired weight ratios. It is denoted as $\mathrm{Li}(\mathrm{G} 4) \mathrm{TFSA}-x \mathrm{PC}$ hereafter, where $x$ represents wt $\%$. Viscosity of the $\mathrm{Li}(\mathrm{G} 4) \mathrm{TFSA}-x \mathrm{PC}$ mixture was evaluated by a digital viscometer (VISCOMATE VM-100A, SEKONIC Corp.).

Self-diffusion coefficients of the constituent species in $\mathrm{Li}(\mathrm{G} 4)$ TFSA- $x$ PC $(x=0$ and 44.5) were measured by pulse-gradient spin-echo NMR (PGSE-NMR) at $298 \mathrm{~K}$. A Brukar Avance 400 spectrometer (Bruker BIOSPIN), equipped with a Diff60 diffusion probe (Bruker BIOSPIN), at a magnetic field of $9.4 \mathrm{~T}$ was used for the NMR measurements, as described elsewhere. ${ }^{22}$ Spectra were collected for the solvents $\mathrm{G} 4$ and $\mathrm{PC}$ for ${ }^{1} \mathrm{H}, \mathrm{TFSA}^{-}$for ${ }^{19} \mathrm{~F}$, and $\mathrm{Li}^{+}$ for ${ }^{7} \mathrm{Li}$, respectively. The self-diffusion coefficients were determined by Hahn's spin-echo pulse sequence based on the Stejskal-Tanner relation, ${ }^{31}$

$$
\frac{S}{S_{0}}=\exp \left\{-\gamma^{2} g^{2} \delta^{2} D\left(\Delta-\frac{\delta}{3}\right)\right\}
$$

where $S, \delta, \gamma$, and $\Delta$ are the spin-echo signal intensity, the length of the gradient pulse, the duration of the field gradient with magnitude, $g$, the gyromagnetic ratio, and the interval between the two gradient pulses, respectively. To evaluate the amounts of ${ }^{1} \mathrm{H}-,{ }^{19} \mathrm{~F}$ - and ${ }^{7} \mathrm{Li}-$ containing species, the repetition times were 1500,1555 , and $1500 \mathrm{~ms}$, respectively, and the maximum gradient values were 200$500,200-800$, and $300-1000 \mathrm{G} \mathrm{cm}^{-1}$, respectively. $\delta$ and $\Delta$ were set to $2.0-2.5 \mathrm{~ms}$ and $30-100 \mathrm{~ms}$, respectively. The obtained exponential PGSE signal attenuations were then fitted by using Eq. (1). The integration times were set to 8-16. Raman spectra (DXR ${ }^{\mathrm{TM}} 2$ Raman Microscope, Thermo Fisher Scientific, Inc.) were taken to evaluate coordination arrangements of solvents to $\mathrm{Li}$ ions with an excitation of the 532-nm line, and stability of $[\mathrm{Li}(\mathrm{G} 4)]^{+}$in the Li(G4)TFSA- $x$ PC mixture was examined. Flash points of Li(G4)TFSA and Li(G4)TFSA-44.5PC were measured according to the JIS $2265 \mathrm{~K}$-based Cleveland open-cup flash-point test (aco-8, Tanaka Scientific, Ltd.).

A self-standing QSE sheet was prepared and used for evaluating ionic conductivity of the QSE. The starting materials, namely, fumed silica powder (Merck KGaK) and $\mathrm{Li}(\mathrm{G} 4) \mathrm{TFSA}$, were mixed at 20:80 volume ratio. Then, the mixture was dispersed in methanol, stirred, and dried at $333 \mathrm{~K}$ to remove the methanol. The resultant QSE powder was further mixed with polytetrafluoroehylene (PTFE) powder (Teflon6-J, Du Pont-Mitsui Fluorochemicals Co., Ltd.) at a weight ratio of 95:5, and pressed to form a transparent sheet with thickness of $200 \mu \mathrm{m}$. The resultant QSE sheet was sandwiched by Li-foils (as electrodes) and placed in a 2032-type coin cell. To evaluate electrolyte resistance, $R$, ac impedance spectra were taken in the frequency range of $1 \mathrm{M}-10 \mathrm{mHz}$ with an input-voltage perturbation of $10 \mathrm{mV}$ (1470E/1400, Solartron Analytical). $R$ was then converted to ionic conductivity, $\sigma$, according to the relationship $\sigma=\frac{1}{R} \frac{L}{A}$, where $L$ and $A$ are respectively QSE thickness and electrode area. To evaluate conductivity of the QSE sheet containing $\mathrm{PC}$, the liquid phase in the QSE, i.e., Li(G4)TFSA, was exchanged by Li(G4)TFSA- $x$ PC. Composition of the liquid phase in the QSE after the liquid-phase exchange was analyzed by NMR, the result of which confirmed that the resultant QSE contained the desired composition of Li(G4)TFSA- $x$ PC.

\subsection{Battery assembly and performance evaluation}

$\mathrm{Li}(\mathrm{Ni}, \mathrm{Co}, \mathrm{Mn}) \mathrm{O}_{2}$ pseudo-ternary oxide (denoted by NCM hereafter) was used for the active material of the positive electrode. For the active material of the negative electrode, graphite was used. A positive-electrode slurry was prepared by mixing NCM with a conductive additive, binder, and $N$-methylpyrrolidone (NMP) at the desired ratios. To prepare a negative-electrode slurry, the graphite negative electrode active material was mixed with styrene-butadiene rubber and carboxymethyl cellulose in water. The resultant slurries were coated on both surfaces of an aluminum current collector and a copper current collector to form positive and negative electrodes, respectively. The formed electrodes were then dried, pressed, and used for the composite positive and negative electrode layers, respectively.

To reduce QSE thickness and subsequently reduce electrolyte resistance of the assembled cells, the QSE slurries containing silica, binder, and NMP were coated on both the positive and negative electrodes. Cross-sectional microstructures of the layered QSE and electrodes were observed by field-emission scanning electron microscopy (FE-SEM, S-4800, Hitachi High-Technologies Corp.). During stacking of the layered QSE and electrodes to form a cell, a short circuit sometimes occurred owing to fracture of cut surfaces and bending. To prevent that short circuit, a separator was placed between the QSE layers of the positive and negative electrodes, as a structure schematically illustrated in Fig. 1(a). The stacked cell (with sized of $21 \times 25 \mathrm{~cm}$ ), containing 26 negative electrodes and 25 positive electrodes, was placed in an aluminum laminate, as shown in the photograph in Fig. 1(b). 
(a)

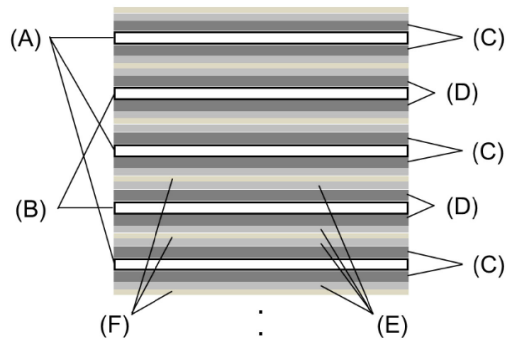

(b)

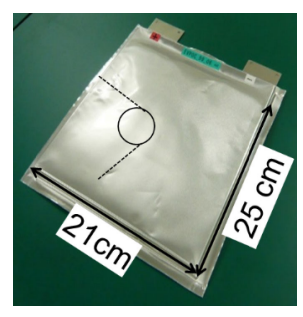

Figure 1. (a) A schematic illustration of the high-energy and highly safe LIB structure: (A) negative-electrode current collector, (B) positive-electrode current collector, (C) negative electrode layer, (D) positive electrode layer, (E) QSE layer and (F) separator. (b) Photograph of the developed 100-Wh-class laminated cell.

An electrolyte containing LiTFSA is known to corrode the current collector of an aluminum positive electrode and thereby causes capacity fading during cycling. ${ }^{32}$ To avoid this phenomenon, a small concentration of a hexafluorophosphate anion $\left(\mathrm{PF}_{6}{ }^{-}\right)$containing additive (which helps to form $\mathrm{AlF}_{3}$ on the surface of the aluminum current collector) was added to the electrolyte. ${ }^{33}$ In addition, to form a stable solid electrolyte interphase (SEI) on the graphite surface, vinylene carbonate (VC) was also added. ${ }^{34}$ A 100Wh-class laminated cell was formed by injecting the mixed liquids, namely, $\mathrm{Li}(\mathrm{G} 4) \mathrm{TFSA}-44.5$ PC containing the two above-mentioned additives, into the stacked LIB and sealing it. The resultant cell contained $261 \mathrm{~g}$ of the electrolyte liquid and $500 \mathrm{~g}$ of solid components.

The above-described $100 \mathrm{Wh}$-class laminated cell was initially charged at a charge rate (c-rate) of $0.005 \mathrm{C}$ for $10 \mathrm{~h}$ and subsequently charged in constant-current (CC) mode at $0.05 \mathrm{C}$ up to an upper cutoff voltage of $4.2 \mathrm{~V}$. At that voltage, charge was continued in constant-voltage $(\mathrm{CV})$ mode until the current decreased to $0.005 \mathrm{C}$. The cell was then discharged in $\mathrm{CC}$ mode at $0.05 \mathrm{C}$ to a lower cutoff voltage of $2.7 \mathrm{~V}$. Discharge-rate capability of the cell was then evaluated after the initial charge and discharge. After that, the cell was charged in $\mathrm{CC}$ mode at $0.2 \mathrm{C}$ up to the upper cut-off voltage of $4.2 \mathrm{~V}$, at which, it was further charged in $\mathrm{CV}$ mode until the current decreased to $0.005 \mathrm{~V}$. Discharge-rate capability in the range of $0.2-$ $3.5 \mathrm{C}$ was then evaluated. To evaluate cycle performance of the cell, the cell was charged at a rate of $0.3-0.03 \mathrm{C}$ in $\mathrm{CC}-\mathrm{CV}$ mode at $4.2 \mathrm{~V}$, and discharged at a rate of $0.3 \mathrm{C}$ in $\mathrm{CC}$ mode to $2.7 \mathrm{~V}$. Discharge capacity at $0.05 \mathrm{C}$ was recorded with an interval of a few-dozen cycles. The retention ratio of discharge capacity was defined by taking the ratio of discharge capacity at $0.05 \mathrm{C}$ in each cycle to that of initial discharge capacity in the first cycle.

\subsection{Nail-penetration test}

The safety of the 100-Wh-class laminated cell assembled with the QSE was evaluated with a nail-penetration test. The cell in fully charged state was sandwiched between two Bakelite plates to ensure thermal insulation. A hole with a 5-mm diameter was drilled at the center of the upper Bakelite plate, and a stainless-steel nail (with a 3$\mathrm{mm}$ diameter and a thermocouple inside its tip) was penetrated into the cell at a rate of $40 \mathrm{~mm} \mathrm{~s}^{-1}$. Another thermocouple was placed at the laminated cell surface and fixed by the Bakelite plate. To examine how the electrolyte composition affects battery safety, the fully charged 100-Wh-class laminated cell using the conventional organic liquid electrolyte, i.e., $1 \mathrm{M} \mathrm{LiPF} 6$ in EC:EMC (33.3:66.7 vol\%), was prepared and also used for the nail penetration test. For the nail-penetration test on this cell, instead of being placed between the QSE layers, the separator was sandwitched by the positive and negative electrodes.

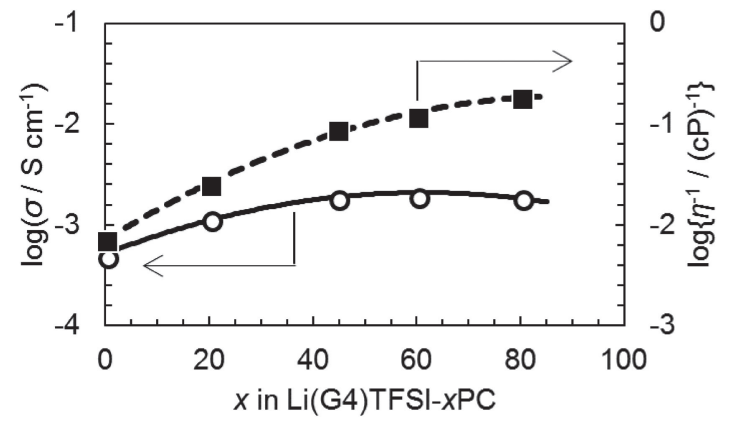

Figure 2. Ionic conductivity of the quasi-solid-state electrolyte containing Li(G4)TFSA- $x$ PC and inverse of viscosity of Li(G4)TFSA- $x$ PC as a function of PC concentration, $x$.

\section{Results \& Discussion}

\subsection{Characterization of quasi-solid-state electrolyte}

Since Li(G4)TFSA contains a high concentration of a LiTFSA, its viscosity is high (i.e., $140 \mathrm{cP}$ ) at room temperature; it thereby compromises lithium-ion transport under current flow and rate capability. Hence, adding a lower-viscosity solvent including PC effectively lowers the viscosity of $\mathrm{Li}(\mathrm{G} 4) \mathrm{TFSA}-x \mathrm{PC}$ and enhances its ion transport. ${ }^{23,24}$ Ionic conductivity, $\sigma$, of the QSE containing $\mathrm{Li}(\mathrm{G} 4)$ TFSA- $x$ PC and inverse of viscosity of the Li(G4)TFSA- $x$ PC mixture are plotted as a function of PC concentration, $x$, in Fig. 2. According to the figure, the inverse of viscosity increases monotonically with increasing $x$, meaning that $\mathrm{PC}$ with a small viscosity of $2.5 \mathrm{cP}$ at $298 \mathrm{~K}$ lowers the viscosity of Li(G4)TFSA$x \mathrm{PC}$. The degree of the decrease of the viscosity is more pronounced in the region where PC concentration is comparatively low. And ionic conductivity exhibits a maximum around $x=60$. It was reported that conductivity is a function of both the diffusion coefficient of the charged species and carrier concentration. ${ }^{35-37}$ Hence, in the region where PC concentration is lower than $60 \%$ weight ratio, conductivity increases with increasing $x$, owing to the enhanced diffusivity of charged species by lowering the viscosity. However, due to the decreased carrier concentration accompanied by dilution of the Li-salt, conductivity started to decrease with further addition of PC when $x$ exceeds $60 \%$. In the region where $40 \leq x \leq 75, \sigma$ exceed $2 \mathrm{mS} \mathrm{cm}^{-1}$. Hence, $x=44.5$ was set for the following evaluations of ion dynamics and battery tests.

To investigate the coordination arrangement of the solvents contained in Li(G4)TFSA- $x$ PC, the self-diffusion coefficients of the diffusing species were evaluated. The self-diffusion coefficients of G4, PC, TFSA, and $\mathrm{Li}$, represented by $D_{\mathrm{G} 4}, D_{\mathrm{PC}}, D_{\mathrm{F}}$ and $D_{\mathrm{Li}}$, respectively, viscosity of the $\mathrm{Li}(\mathrm{G} 4) \mathrm{TFSA}-x \mathrm{PC}(x=0$ and 44.5$)$ mixture, and ionic conductivity of the QSE containing Li(G4)TFSA$x \mathrm{PC}$ are summarized in Table 1 . For $x=0, \log \left(D_{\mathrm{G} 4} / \mathrm{m}^{2} \mathrm{~s}^{-1}\right)$, $\log \left(D_{\mathrm{F}} / \mathrm{m}^{2} \mathrm{~s}^{-1}\right)$, and $\log \left(D_{\mathrm{Li}} / \mathrm{m}^{2} \mathrm{~s}^{-1}\right)$ are -11.1 at $298 \mathrm{~K}$, which is consistent with values in the literature. ${ }^{20,21}$ The ratio of the selfdiffusion coefficient of $\mathrm{G} 4$ to that of $\mathrm{Li}, D_{\mathrm{G} 4} / D_{\mathrm{Li}}$, is 0.97 , suggesting that G4 is essentially coordinated with $\mathrm{Li}$ ions, forming $\mathrm{Li}(\mathrm{G} 4)$ TFSA, and a very low concentration of free G4 stays. ${ }^{23,24}$ For $x=44.5$, ionic conductivity of the QSE as well as the self-diffusion coefficients of $\mathrm{G} 4, \mathrm{~F}$, and $\mathrm{Li}$ are enhanced by the addition of lowerviscosity PC. $\log \left(D_{\mathrm{G} 4} / \mathrm{m}^{2} \mathrm{~s}^{-1}\right), \log \left(D_{\mathrm{F}} / \mathrm{m}^{2} \mathrm{~s}^{-1}\right)$, and $\log \left(D_{\mathrm{Li}} / \mathrm{m}^{2} \mathrm{~s}^{-1}\right)$ are similar, namely, -10.0 . And $D_{\mathrm{G} 4} / D_{\mathrm{Li}}$ is 1.17 , suggesting that free G4 is formed by a ligand exchange with PC in the Li(G4)TFSA$x \mathrm{PC}$ mixture, as discussed elsewhere. ${ }^{23,24}$

Stability of $[\mathrm{Li}(\mathrm{G} 4)]^{+}$in $\mathrm{Li}(\mathrm{G} 4) \mathrm{TFSA}-x \mathrm{PC}$ was further investigated by using Raman spectroscopy. Raman spectra of Li(G4)TFSA- $x$ PC at various $x$ over $900-800 \mathrm{~cm}^{-1}$ are shown in Fig. 3. In this figure, intensity of the spectra for $x=20-80$ are normalized by 
Table 1. Summary of ionic conductivity of the quasi-solid-state electrolyte containing Li(G4)TFSA- $x$ PC, $\sigma$, viscosity of Li(G4)TFSA- $x$ PC, $\eta$, and self-diffusion coefficients of G4, $D_{\mathrm{G} 4}, \mathrm{PC}, D_{\mathrm{PC}}, \mathrm{F}, D_{\mathrm{F}}$, and $\mathrm{Li}, D_{\mathrm{Li}}$ at $298 \mathrm{~K}$.

\begin{tabular}{ccccccc}
\hline$X$ & $\log \left(D_{\mathrm{G} 4} / \mathrm{m}^{2} \mathrm{~s}^{-1}\right)$ & $\log \left(D_{\mathrm{PC}} / \mathrm{m}^{2} \mathrm{~s}^{-1}\right)$ & $\log \left(D_{\mathrm{F}} / \mathrm{m}^{2} \mathrm{~s}^{-1}\right)$ & $\log \left(D_{\mathrm{Li}} / \mathrm{m}^{2} \mathrm{~s}^{-1}\right)$ & $\sigma / \mathrm{mS} \mathrm{cm}^{-1}$ & $\eta / \mathrm{cP}$ \\
\hline 0 & -11.1 & - & -11.1 & -11.1 & 0.5 & 140 \\
44.5 & -10.0 & -9.7 & -10.0 & -10.0 & 1.9 & 11.2 \\
\hline
\end{tabular}

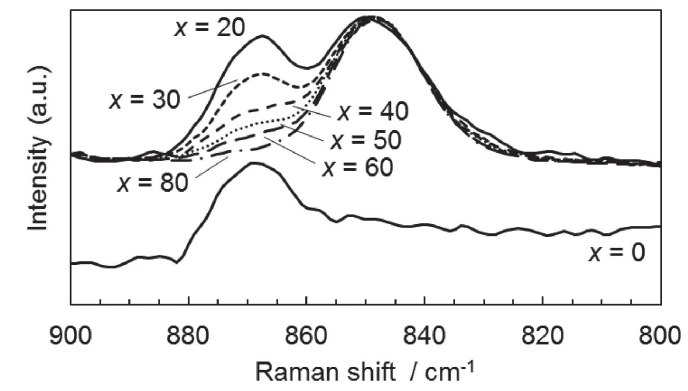

Figure 3. Raman spectra for Li(G4)TFSA- $x$ PC. For $x=20-80$, spectrum intensities were normalized by the intensity at around $850 \mathrm{~cm}^{-1}$ (maximum intensity).

peak intensity appearing at around $850 \mathrm{~cm}^{-1}$. For $x=0$, a band, which is originated from a complexation of G4 with Li-ions, appears at around $870 \mathrm{~cm}^{-1} \cdot 23,34$ Then, a new band around $850 \mathrm{~cm}^{-1}$, originated from free $\mathrm{G} 4$, appears with addition of $\mathrm{PC}$, and the intensity of a band around $870 \mathrm{~cm}^{-1}$ becomes less pronounced with increasing concentration of $\mathrm{PC}, x$. This result suggests that a ligand is exchanged from $\mathrm{G} 4$ to $\mathrm{PC}$ in the $\mathrm{Li}(\mathrm{G} 4)$ TFSA- $x \mathrm{PC}$ mixture; thereby, the concentration of free G4 is increased by increasing $x,{ }^{23,38}$ and that result agrees well with the results concerning the selfdiffusion coefficients as discussed earlier.

Li(G4)TFSA has a flash point of $451 \mathrm{~K}$, which is higher than that G4 solvents, i.e., $414 \mathrm{~K}^{28}$ This fact implies that forming an equimolar complex of lithium salt and ether also enhances battery safety. Since PC, which has a flash point of $405 \mathrm{~K},{ }^{45}$ was used in the present study as a diluting solvent, the flash point of Li(G4)TFSA44.5PC remained high $(415 \mathrm{~K})$, which is higher than that of the conventional electrolyte liquid, i.e., 293-303 K..$^{29,30}$

PC solvent promotes co-intercalation of the solvated species into the graphite negative electrode and its reduction during battery operation. ${ }^{39}$ In addition, excess glyme solvent contained in SILs promotes co-intercalation. ${ }^{40}$ These co-intercalations can interfere with repeated battery operation. In fact, our preliminary half-cell test on the graphite negative electrode, comprised of Li(G4)TFSA44.5PC, revealed remarkable capacity fading within a few dozen cycles. Considering these results, we decided to introduce $\mathrm{VC}$ in this study as an SEI former at the graphite surface in order to ensure repeated battery operation. ${ }^{34}$ After optimizing $\mathrm{VC}$ concentration based on the results of a half-cell test on the graphite negative electrode, we used the QSE containing the Li(G4)TFSA-PC mixture for assembling the 100-Wh-class laminated cell.

\subsection{Battery performance}

After optimizing the QSE slurry, compositions, and coating conditions, we formed a uniform QSE layer with $20-\mu \mathrm{m}$ thickness at the non-uniform surfaces of both the composite positive and negative electrodes, as SEM images shown in Fig. 4.

We developed the electrolyte comprised of the ionic-conductivity-optimized $\mathrm{Li}(\mathrm{G} 4)$ TFSA-44.5PC mixed with VC (as the SEI former at the graphite surface) and $\mathrm{PF}_{6}$-based additives (as $\mathrm{LiF}_{3}$ former at the positive-electrode aluminum-current-collector surface) for suppressing its corrosion. ${ }^{33}$ In the preliminary battery-perform-
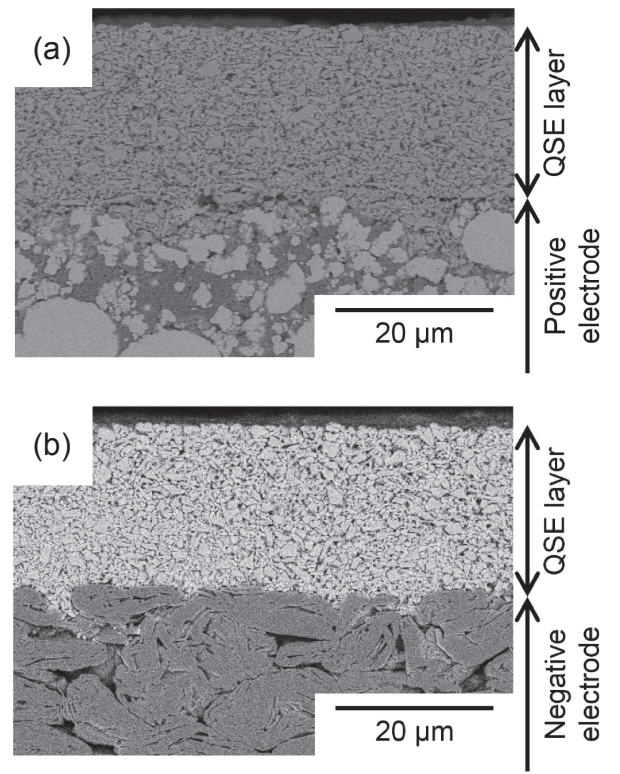

Figure 4. Cross-sectional back-scattered SEM images: (a) positive electrode and (b) negative electrode. These electrodes are used for assembling the 100-Wh-class laminated cell.

ance test, quick charging in the initial cycle compromised coulombic efficiency (defined by the ratio of discharge capacity to charge capacity), and a side-reaction accompanies by generations of gases. For this reason, we speculate that the quick initial charge caused a porous and non-uniform SEI layer to form at the graphite surface, and that layer causes the side-reaction. When the cell was charged very slowly, e.g., at $0.005 \mathrm{C}$, at very beginning of the initial-charge step, the initial coulombic efficiency was enhanced, and the quantity of the gas generation was suppressed. This result suggests that the very slow charge allows for the formation of a preferential and uniform SEI at the graphite surface, and the formed SEI hinders the side-reaction of the electrolyte.

The initial charge-discharge profile of the $100 \mathrm{Wh}$-class laminated cell is shown in Fig. 5(a). To form the preferential SEI, the cell was charged at $0.005 \mathrm{C}$ until the capacity reached $1.7 \mathrm{Ah}(10 \mathrm{~h}$ required). After that charge, the cell was charged at $0.05 \mathrm{C}$ up to the upper cutoff voltage of $4.2 \mathrm{~V}$, and the voltage remained at $4.2 \mathrm{~V}$ until the current decreased to $0.005 \mathrm{C}$. The cell was then discharged at $0.05 \mathrm{C}$ until the voltage decreased to $2.7 \mathrm{~V}$. The cell recorded initial charge capacity of $42 \mathrm{Ah}$ and initial discharge capacity of $32 \mathrm{Ah}$, and its coulombic efficiency was high $(76 \%)$. Since average reaction voltage is $3.67 \mathrm{~V}$, discharge energy was calculated to be $115 \mathrm{Wh}$. Then, based on the cell geometry, energy density of the cell was calculated to be $363 \mathrm{Wh} \mathrm{L}^{-1}$ and $135 \mathrm{Wh} \mathrm{kg}^{-1}$.

Discharge-rate capability of the 100 -Wh-class laminated cell was evaluated. Discharge capacity is plotted as a function of discharge rate in Fig. 5(b). In the region where c-rate is less than $1 \mathrm{C}$, discharge capacity is greater than $30 \mathrm{Ah}$ and almost independent of discharge rate. The cell still exhibited discharge capacity of $26 \mathrm{Ah}$ at $2 \mathrm{C}$. This high discharge-rate performance is a result of mixing PC with $\mathrm{Li}(\mathrm{G} 4)$ TFSA. That is, decreasing viscosity enhanced lithium- 

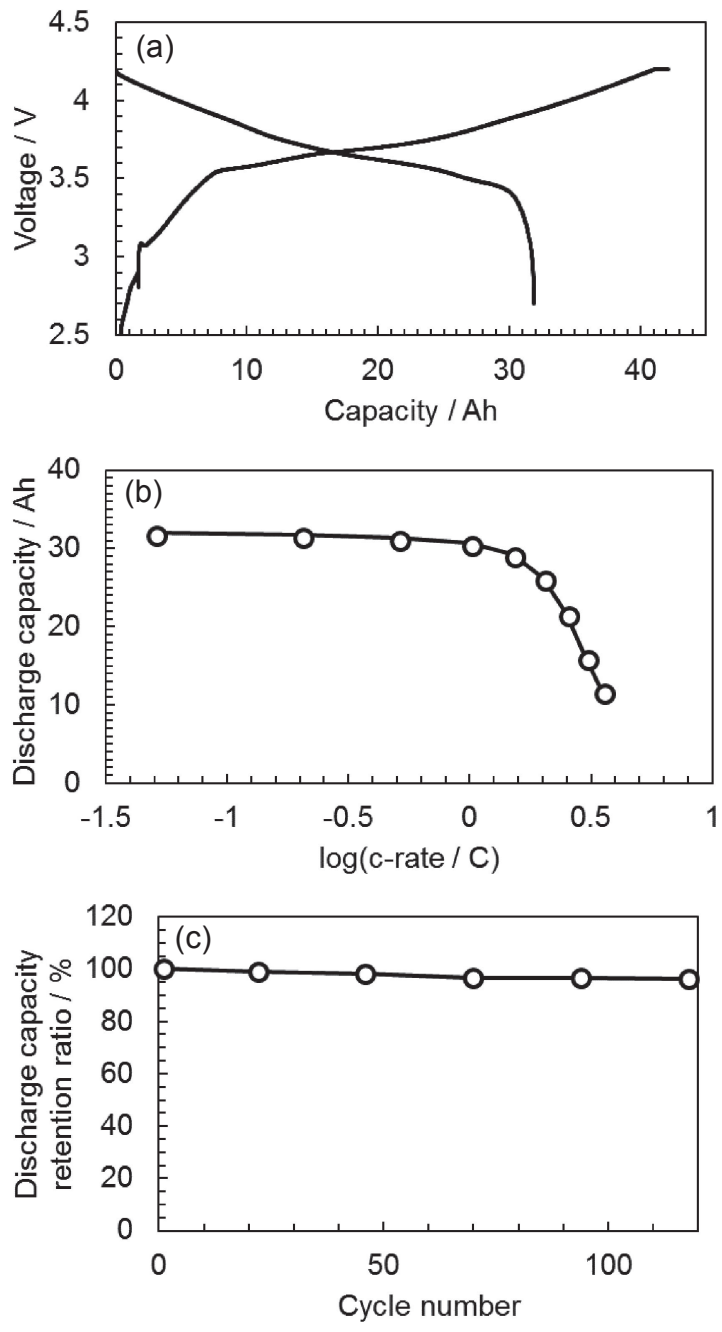

Figure 5. Battery performance of the 100 -Wh-class laminated cell: (a) typical initial charge-discharge curves, (b) rate capability, and (c) cycle performance.

ion diffusivity in the QSE. In addition, accompanied by the dilution with the PC solvent, the increased resistance due to concentration polarization under high discharge rate was mitigated.

Repeated operation of the 100 -Wh-class laminated cell was enabled by addition of the SEI former at the graphite surface and an $\mathrm{AlF}_{3}$ former on the aluminum-current-collector surface, which hinder the side reaction during repeated battery operation over a long cycle life. Discharge-capacity retention ratio is plotted as a function of cycle number in Fig. 5(c). According to the figure, the cell retained discharge capacity of nearly $96 \%$ at the 118 th cycle.
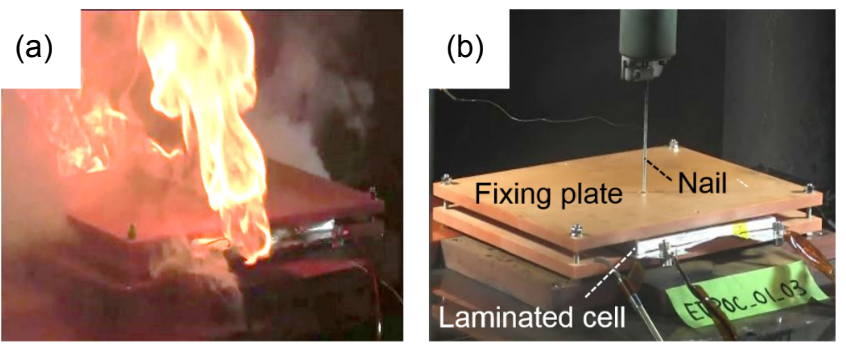

Figure 6. Nail-penetration-test results: 100-Wh-class laminated cell using (a) conventional organic liquid electrolyte, $1 \mathrm{M} \mathrm{LiPF}_{6}$ in EC:EMC (33.3:66.7 vol\%), and (b) quasi-solid-state electrolyte, comprised of Li(G4)TFSA-44.5PC.

\subsection{Nail-penetration test}

When a metallic nail penetrates a LIB under charged state, a short circuit occurs via the nail, and joule heat is generated. The generated joule heat triggers oxygen release from the positive electrode, and that oxygen feeds continuous combustion of the electrolyte, so called "thermal runaway". ${ }^{41}$ For the fully charged 100-Wh-class laminated cell containing the conventional organic liquid electrolyte, i.e., $1 \mathrm{M} \mathrm{L^{2PF }} 6$ in EC:EMC (33.3:66.7 vol\%), typical thermal runaway took place as shown in the photograph in Fig. 6(a). That is, joule heat was generated after the nail penetrated the cell and, subsequently, fire and smoke appeared. Soon after the nail penetration, the temperatures of the tip of the nail and in the cell were increased to $747 \mathrm{~K}$ and $817 \mathrm{~K}$, respectively. Accompanied by this temperature increase, cell voltage dropped to $0 \mathrm{~V}$, as shown by the temperature and voltage profiles in Fig. 7(a). On the other hand, when the fully charged 100 -Wh-class laminated cell using the QSE, comprised of Li(G4)TFSA-44.5PC, was subjected to the same test, fire and smoke were not generated, as shown in Fig. 6(b), and the temperatures of a tip of the nail and the cell remained low, i.e., $365 \mathrm{~K}$ and $324 \mathrm{~K}$, respectively, as shown in Fig. 7(b). Despite the short circuit via the nail, cell voltage remained high, i.e., $4.03 \mathrm{~V}$ (compared with $4.2 \mathrm{~V}$ before the nail-penetration test). These results suggest that the temperature increase and voltage drop due to the short circuit are very limited in the case of the newly developed LIB. This result implies that although some joule heat was generated, subsequent combustion was hindered by the QSE layers; thereby, thermal runaway did not take place.

There might be an argument that the battery performances, including capacity and rate capability, are the factors that influence the nail penetration test results. The difference of these performances between the LIBs comprised of the QSE and the organic liquid electrolyte were small for our experiments. The capacities of the LIBs using the QSE and the organic liquid electrolyte were $32 \mathrm{Ah}$ and $37 \mathrm{Ah}$, respectively. The discharge capacities at $2 \mathrm{C}$ (rate capability) of the QSE-based LIB and the organic liquid electrolyte-
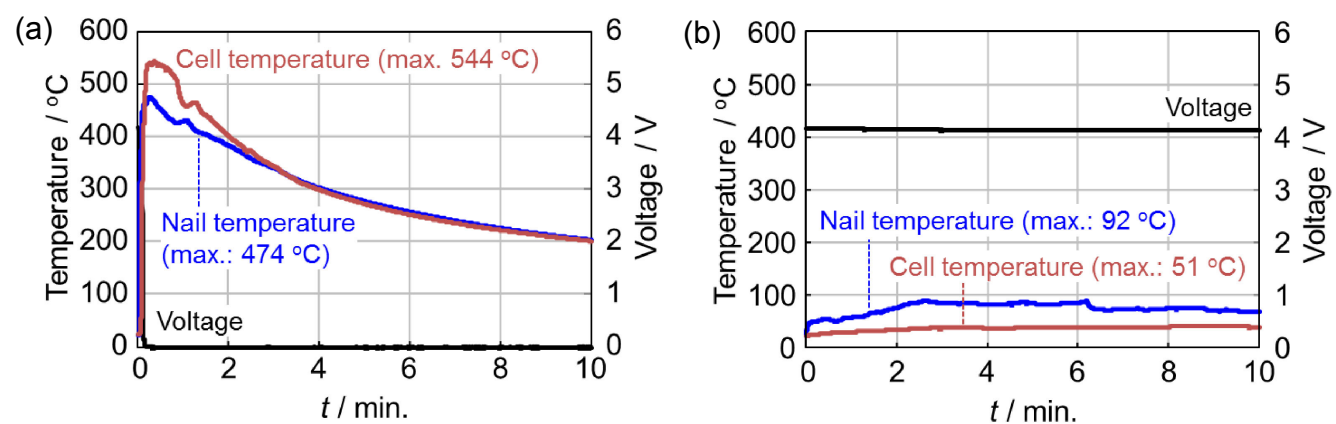

Figure 7. Temperature and voltage profiles after the nail-penetration tests for the 100-Wh-class laminated cell using: (a) conventional organic liquid electrolyte, $1 \mathrm{M} \mathrm{LiPF}_{6}$ in EC:EMC (33.3:66.7 vol\%), and (b) quasi-solid-state electrolyte, comprised of Li(G4)TFSA-44.5PC. 
based LIB were $26 \mathrm{Ah}$ and $30 \mathrm{Ah}$, respectively. For quantitatively clarifying the safety limit of our battery configuration, we are investigating how the battery performance as parameters affects the safety of the LIBs assembled with the QSE layer. The results may appear and further discussion will be carried out in a separeated paper.

As described above, at least for a nail-penetration test, our highenergy $100 \mathrm{Wh}$-class LIB with energy density of $363 \mathrm{Wh} \mathrm{L}^{-1}$ using the QSE demonstrated higher level of safety compared to the cell using the conventional organic-liquid electrolyte. One of the possible reasons for passing the nail-penetration test, as shown in Fig. 6(b), is considered to be the high flash point of the liquid phase; namely, organic-liquid electrolyte, $\mathrm{LiPF}_{6}$ in EC:EMC, has a flash point of $293-303 \mathrm{~K},{ }^{28,29}$ while $\mathrm{Li}(\mathrm{G} 4) \mathrm{TFSA}-\mathrm{PC}$ has one of $415 \mathrm{~K}$ (as explained in Section 3.1). That result agrees with that revealed by ignition tests showing the safety of a mixture of roomtemperature ionic liquid and solvents. ${ }^{26,42}$ Another possible reason is the existence of the QSE layer formed by oxide particles and binder, which can exist and aviod a direct contact between the nail and electrodes during the nail penetration, ${ }^{43}$ and thereby the voltage drop during the test is mitigated, as the voltage profile shown in Fig. 7(b). In that case, the quantity of joule heat generated by the nail penetration, which triggers thermal runaway, should be affected by the microstructure and composition of the QSE layer existing between the positive and negative electrodes. Hence, optimizing not only the liquid phase but also the QSE layer would be advantageous for further ensuring the safety of a LIB. Specifically, it has been reported that rate of joule-heat generation by a short circuit is faster (on a logarithmic scale) than that of the combustion reaction of the electrolyte, and the combustion of the electrolyte is triggered by the joule-heat generation. ${ }^{41}$ When the quantity of joule-heat generation is lowered by the LIB materials and modifications to the microstructure of the QSE layer, it is possible to avoid occurrence of the subsequent combustion reaction and, thereby, thermal runaway is hindered. To fully understand the mechanism for ensuring LIB safety by the nail-penetration test, further investigation is needed.

As described above, high energy density, good rate capability and long cycle life were successfully demonstrated for our newly developed LIB. In addition, our battery passed the nail penetration test owing to the existence of the QSE layer. This means that use of the QSE layer is essential for satisfying both electrochemical battery performance and safety in our battery configuration. Based on these experimental results, it is considered that two types of electrolyte liquid stay in our battery configuration. One is in the QSE layer that forms ion transport paths in the electrolyte layer. As discussed in the Introduction section, the electrolyte liquid is quasi-solidified just beneath the silica particle surface, owing to the strong interaction between the silica surface and electrolyte liquid containing the solvate ionic liquid. ${ }^{10-19}$ Then, the QSE also contains electrolyte liquid free from that interaction. As discussed above the QSE layer likely contributed to enhancing battery safety in the nail penetration test. The other electrolyte liquid is in the positive and negative electrode layers that were introduced by the electrolyte liquid injection. This type of electrolyte liquid allows for forming ion transport paths in the electrode layers, and hence excellent battery performance is realized.

A battery pack built with the developed 100-Wh-class laminated cells could be a power source of an electric vehicle (EV). A module was configured with six cells in parallel, and a battery pack containing 14 of those modules in series was then built. Eighty-four cells were used for building the battery pack, so the resulting battery pack had an energy of $10 \mathrm{kWh}$. Then, the $10-\mathrm{kWh}$ battery pack was installed in a commercially available compact EV, instead of its original battery pack. A controlling unit for regenerative braking energy was also developed and used in the battery pack. The EV containing the battery pack allowed for driving in areas of the Hitachi Research Institute, with a maximum speed of $35 \mathrm{~km} \mathrm{~h}^{-1}$. During this driving, maximum charge current reached to $78 \mathrm{~A}$ (corresponding to $0.4 \mathrm{C}$ ) and discharge current was $123 \mathrm{~A}(0.6 \mathrm{C})$. Owing to the enhanced LIB volumetric energy density and optimized battery-pack design, we could reduce total volume of the battery pack by two-thirds. This result suggests that ensuring battery safety is crucial and beneficial for enhancing energy density of the battery pack.

In addition to clarifying the mechanism that allows the LIB to pass the nail-penetration test, we are currently investigating the safety of the developed cell based on different safety tests, including crushing and overcharging. ${ }^{44}$ By combining the knowledge obtained from these safety tests, we are striving to develop essentially safe, high-energy, and high-energy-density LIBs in future.

\section{Conclusions}

A high-energy and high-safety 100-Wh-class laminated LIB was developed. As for the assembly of this LIB, SIL, Li(G4)TFSA, diluted by PC, was used, and the resultant liquid phase was quasisolidified by mixing with silica particles. The chemical composition, including mixing ratio of $\mathrm{Li}(\mathrm{G} 4) \mathrm{TFSA}$ and PC, and additives for forming the SEI at the graphite surface and $\mathrm{AlF}_{3}$ at the aluminum current collecto was optimized for enhancing battery performance and safety. The developed LIB exhibited initial discharge capacity of $32 \mathrm{Ah}$ at $0.05 \mathrm{C}$. Its discharge capacity at $2 \mathrm{C}$ stayed high, i.e., 26.2 Ah. And the ratio of its discharge capacity at the 118th cycle to its initial discharge capacity remained high, i.e., 96\%. By a nailpenetration test, higher safety of the developed 100-Wh-class LIB compared to the LIB using a conventional organic-liquid electrolyte was demonstrated. Currently, we are further investigating the safety mechanism that allows the LIB to pass the nail-penetration test. In addition, we are extending our work on other safety tests, including crushing and overcharging. On the basis of the knowledge obtained from the results of various safety tests, we are striving to develop essentially safe, high-energy and high-energy-density LIBs that meet requirements for large-scale applications in terms of battery performance and cost competitiveness in future.

\section{References}

1. D. H. Doughty and E. P. Roth, Electrochem. Soc. Interface, 21, 37 (2012)

2. J. B. Goodenough and K.-S. Park, J. Am. Chem. Soc., 135, 1167 (2013).

3. K. Takada, Acta Mater., 61, 759 (2013).

4. N. Kamaya, K. Homma, Y. Yamakawa, M. Hirayama, R. Konno, M. Yonemura, T. Kamiyama, Y. Kato, S. Hama, K. Kawamoto, and A. Mitsui, Nat. Mater., 10, 682 (2011).

5. Y. Kato, S. Hori, T. Saito, K. Suzuki, M. Hirayama, A. Mitsui, M. Yonemura, H. Iba, and R. Kanno, Nat. Energy, 1, 16030 (2016).

6. N. Ohta, K. Takada, L. Zhang, R. Ma, M. Osada, and T. Sasaki, Adv. Mater, 18, 2226 (2006).

7. A. Sakuda, A. Hayashi, and M. Tatsumisago, Chem. Mater., 22, 949 (2010).

8. M. Galinski, A. Lewandowski, and I. Stepniak, Electrochim. Acta, 51, 5567 (2006).

9. M. Watanabe, M. L. Thomas, S. Zhang, K. Ueno, T. Yasuda, and K. Dokko, Chem. Rev, 117, 7190 (2017).

10. M. Mezger, H. Schroder, H. Reichert, S. Schramm, J. S. Okasinski, S. Schoder, V. Honkimaki, M. Deutsch, B. M. Ocko, J. Ralstron, M. Rohwerder, M. Stratmann, and H. Dosch, Science, 322, 424 (2008).

11. K. Ueno, M. Kasuya, M. Watanabe, M. Mizukami, and K. Kurihara, Phys. Chem. Chem. Phys., 12, 4066 (2010).

12. T. Fukushima, A. Kosaka, Y. Ishimura, T. Yamamoto, T. Takigawa, N. Ishii, and T. Aida, Science, 300, 2072 (2003).

13. S. Shimano, H. Zhou, and I. Honma, Chem. Mater, 19, 5216 (2007).

14. T. Katakabe, R. Kawano, and M. Watanabe, Electrochem. Solid-State Lett., 10, F23 (2007).

15. T. Katakabe, T. Kaneko, M. Watanabe, T. Fukushima, and T. Aida, J. Electrochem. Soc., 152, A1913 (2005).

16. S. Ito, A. Unemoto, H. Ogawa, T. Tomai, and I. Honma, J. Power Sources, 208, 271 (2012). 
17. H. Ogawa, A. Unemoto, and I. Honma, Electrochemistry, 80, 765 (2012).

18. A. Unemoto, H. Ogawa, S. Ito, and I. Honma, J. Electrochem. Soc., 160, A138 (2013).

19. A. Unemoto, H. Ogawa, S. Ito, and I. Honma, J. Electrochem. Soc., 160, A138 (2013).

20. T. Tamura, K. Yoshida, T. Hashida, M. Tsuchiya, M. Nakamura, Y. Kazue, N. Tachikawa, K. Dokko, and M. Watanabe, Chem. Lett., 39, 753 (2010).

21. K. Yoshida, M. Nakamura, Y. Kazue, N. Tachikawa, S. Tsuzuki, S. Seki, K. Dokko, and M. Watanabe, J. Am. Chem. Soc., 133, 13121 (2011).

22. A. Unemoto, T. Matsuo, H. Ogawa, Y. Gambe, and I. Honma, J. Power Sources, 244, 354 (2013).

23. K. Ueno, J. Murai, K. Ikeda, S. Tsuzuki, M. Tsuchiya, R. Tatara, T. Mandai, Y. Umebayashi, K. Dokko, and M. Watanabe, J. Phys. Chem. C, 120, 15792 (2016).

24. K. Ueno, J. Murai, H. Moon, K. Dokko, and M. Watanabe, J. Electrochem. Soc., 164, A6088 (2017).

25. K. Dokko, N. Tachikawa, K. Yamauchi, M. Tsuchiya, A. Yamazaki, E. Takashima, J.-W. Park, K. Ueno, S. Seki, N. Serizawa, and M. Watanabe, J. Electrochem. Soc., 160, A1304 (2013).

26. R.-S. Kuhnel, N. Bockenfeld, S. Passerini, M. Winter, and A. Balducci, Electrochim. Acta, 56, 4092 (2011).

27. A. Ponrouch, D. Monti, A. Boschin, B. Steen, P. Johansson, and M. R. Palacin, J. Mater. Chem. A, 3, 22 (2015).

28. F. Chretien, J. Jones, C. Damas, D. Lemordant, P. Willmann, and M. Anouti, J. Power Sources, 248, 969 (2014).

29. M. Otsuki, T. Ogino, and K. Amine, ECS Trans., 1, 13 (2006).

30. M. Otsuki and T. Oguno, Lithium-Ion Batteries (Eds. M. Yoshio, R. J. Brodd, and A. Kozawa), Springer Nature, Switzerland, Chap. 13 (2009).
31. E. O. Stejskal and J. E. Tanner, J. Chem. Phys., 42, 288 (1965).

32. L. J. Krause, W. Lamanna, J. Summerfield, M. Engle, G. Korba, R. Koch, and R. Atanasoski, J. Power Sources, 68, 320 (1997).

33. N. Iwayasu, WO2018/030150, Feb. 15, 2018.

34. D. Aurbach, K. Gamolsky, B. Markovsky, Y. Gofer, M. Schmidt, and U. Heider, Electrochim. Acta, 47, 1423 (2002).

35. R. Hagiwara and Y. Ito, J. Fluorine Chem., 105, 221 (2000)

36. H. Tokuda, K. Hayamizu, K. Ishii, M. A. B. H. Susan, and M. Watanabe, J. Phys. Chem. B, 109, 6103 (2005).

37. A. Noda, K. Hayamizu, and M. Watanabe, J. Phys. Chem. B, 105, 4603 (2001).

38. D. Brouillette, D. E. Irish, N. J. Taylor, G. Perron, M. Odziemkowski, and J. E. Denoyers, Phys. Chem. Chem. Phys., 4, 6063 (2002).

39. D. Aurbach, M. Koltypin, and H. Teller, Langmuir, 18, 9000 (2002).

40. H. Moon, R. Tatara, T. Mandai, K. Ueno, K. Yoshida, N. Tachikawa, T. Yasuda, K. Dokko, and M. Watanabe, J. Phys. Chem. C, 118, 20246 (2014).

41. C.-H. Doh, D.-H. Kim, H.-S. Kim, H.-M. Shin, Y.-D. Jeong, S.-I. Moon, B.-S. Jin, S. W. Eom, H.-S. Kim, K.-W. Kim, D.-H. Oh, and A. Veluchamy, J. Power Sources, 175, 881 (2008).

42. A. Guerfi, M. Dontigny, P. Charest, M. Petitclerc, M. Lagace, A. Vijh, and K. Zaghib, J. Power Sources, 195, 845 (2010).

43. Y. Deng, X. Song, Z. Ma, X. Zhang, D. Shu, and J. Nan, Electrochim. Acta, 212, 416 (2016).

44. Q. Wang, P. Ping, X. Zhao, G. Chu, J. Sun, and C. Chen, J. Power Sources, 208, 210 (2012).

45. K.-C. Moller, T. Hodal, W. K. Appel, M. Winter, and J. O. Besenhard, J. Power Sources, 97-98, 595 (2001). 